\title{
Exosomes as "translational" cancer promoter organelles
}

\author{
Isabella Panfoli \\ University of Genova, School of Medical and Pharmaceutical Sciences, DIFAR-Biochemistry Lab., Viale Benedetto XV, 316132 Genova, Italy \\ Correspondence to: Isabella Panfoli, MD. University of Genova, DIFAR-Biochemistry Lab., Vale Benedetto XV, 316132 Genova, Italy. \\ Email: Isabella.Panfoli@unige.it.
}

Submitted Jun 18, 2016. Accepted for publication Jun 29, 2016.

doi: 10.21037/tcr.2016.08.08

View this article at: http://dx.doi.org/10.21037/tcr.2016.08.08

The term Exosome has come into use to define nanovesicles contained in multivesicular endosomes (MVE), secreted by fusion of MVE with the plasma membrane (1-3). Exosomes are secreted in vivo by almost any cell type and can be isolated from body fluids (4-6). Indeed, circulating vesicles account for both exosomes and microvesicles (MVs), which can be purified by various purification methods and fully discriminated according to their shape, size and CDmarkers $(7,8)$. Due to their protein and RNA content, once internalized, exosomes have the potential to act as "translational" organelles, altering the expression pattern of recipient cells, their growth, and fate. In fact, exosomes are involved in many of physio-pathological processes, thereby including cancer (9).

The work recently published by Franzen $e t a l$. in The Fournal of Urology (10) examined the role of exosomes in intercellular communication and their potential as noninvasive cancer biomarker source to assess disease and its progression, or vehicles for therapeutic delivery in urologic cancer (11). The need for a predictor of malignancy is universally recognized. This is particularly true in the case of early tumors. There is evidence that cancer-derived exosomes contribute to tumor progression and metastasis (12). Tumor cells produce exosomes, emerging as a potential for the early detection or therapy of human cancer (13).

Human urinary exosomal proteome has been extensively studied (7). Urinary exosomes come from every cell type of the urinary tract, kidney and prostate (14-16). There is increasing interest in urinary exosomes, due to their ability to carry information specific of the tissue of origin. I am positive about the idea that exosomes, which can be easily isolated from human urine by minimally invasive techniques, can allow to detect biomarkers in patients with urogenital cancer, with a wealth of applications in therapy and diagnosis, as we have already observed (17).
Bladder cancer is one example, as it represents a serious health problem, (about $8 \%$ of all human malignancies), still burdened by a high percentage of relapse (18).

However, I am sceptic to the idea that exosomes can be used in therapeutics as RNA or therapy delivery vector to target cancer cells. Surely, RNA would be protected by the membrane from degradation. Nevertheless, it seems that we still know too little about the surface proteins of exosomes. We have shown that among the exosomal surface proteins there are the respiratory chains and $\mathrm{F}_{1} \mathrm{~F}_{\mathrm{o}}$-ATP synthase, conducting an oxidative phosphorylation $(19,20)$. Before seeing the contents of a package, its envelope must be opened. Similarly, before the RNA content of an exosome complex is shed, and it can affect the cell expression pattern, its surface proteins will have interacted with the cytosol. We have reported the proteome analysis of urinary exosomes, studied by Orbitrap mass spectrometry, compared to urinoma (20). Cytoscape software analysis of the data elucidated the enriched presence in urinary exosomes of proteins clustered to aerobic metabolism. Moreover, functional experiments showed that urinary exosomes carry out oxidative phosphorylation. The same applies to exosomes derived from human umbilical cord mesenchymal cells (MSC), which are able to conduct an aerobic metabolism (19). Such emerging metabolic function for both human MSC and urinary exosomes should not be ignored. It appears consistent with the report that exosomes can transfer the aerobic metabolism capacity to profoundly hypo-metabolic cells in less than one hour (21). This dramatic effect overturned the fate of doomed cells long before any transcriptional event can have occurred. In case of tumor cells, this may not be desirable. For example it was found that various concentrations of exosomes purified from the supernatants of human bladder cancer T24 cell cultures induce the proliferation and decrease the rates of 
apoptosis (22). Having the observations been limited to late events, posing ATP availability as a prerequisite (viability assay of at $72 \mathrm{hr}$, Annexin $\mathrm{V}$ and transcription factors activity), an involvement of early metabolic effect cannot be excluded. In fact, it was also found that bladder cancer exosomes can cause urothelial cells to undergo epithelial-mesenchymal transition (EMT). Authors treated primary urothelial cells with the exosomes isolated mRNA and evaluated the expression of several mesenchymal genes necessarily after a 4- and 6-h time to reveal newly transcribed mRNAs (23). While it is known that the EMT induces invasive properties in epithelial tumors, very little is known about EMT-induced metabolic changes. A study on HER2-positive BT-474 breast cancer cells showed enhanced aerobic metabolism, along with the overexpression of specific glucose transporters (24).

A method to be used clinically should be simple, costeffective and minimally invasive, which exosome collection from urine appears to be. In conclusion, I am optimistic that further studies about the potential for exosomes will help in diagnosis, treatment and prognosis assessment of urinary tract cancer. However, several unanswered questions regarding the metabolic potential of exosomes remain. In this context, improved knowledge about the metabolism of exosomes are needed.

\section{Acknowledgements}

None.

\section{Footnote}

Provenance: This is a Guest Editorial commissioned by Section Editor Peng Zhang (Department of Urology, Union Hospital, Tongji Medical College, Huazhong University of Science and Technology, Wuhan, China).

Conflicts of Interest: The author has no conflicts of interest to declare.

Comment on: Franzen CA, Blackwell RH, Foreman KE, et al. Urinary Exosomes: The Potential for Biomarker Utility, Intercellular Signaling and Therapeutics in Urological Malignancy. J Urol 2016;195:1331-9.

\section{References}

1. Vlassov AV, Magdaleno S, Setterquist R, et al. Exosomes: current knowledge of their composition, biological functions, and diagnostic and therapeutic potentials. Biochim Biophys Acta 2012;1820:940-8.

2. Mathivanan S, Ji H, Simpson RJ. Exosomes: extracellular organelles important in intercellular communication. J Proteomics 2010;73:1907-20.

3. Pant $\mathrm{S}$, Hilton H, Burczynski ME. The multifaceted exosome: biogenesis, role in normal and aberrant cellular function, and frontiers for pharmacological and biomarker opportunities. Biochem Pharmacol 2012;83:1484-94.

4. Wang D, Sun W. Urinary extracellular microvesicles: isolation methods and prospects for urinary proteome. Proteomics 2014;14:1922-32.

5. Lässer C, Alikhani VS, Ekström K, et al. Human saliva, plasma and breast milk exosomes contain RNA: uptake by macrophages. J Transl Med 2011;9:9.

6. Pisitkun T, Shen RF, Knepper MA. Identification and proteomic profiling of exosomes in human urine. Proc Natl Acad Sci U S A 2004;101:13368-73.

7. Conde-Vancells J, Rodriguez-Suarez E, Embade N, et al. Characterization and comprehensive proteome profiling of exosomes secreted by hepatocytes. J Proteome Res 2008;7:5157-66.

8. Pan BT, Teng K, Wu C, et al. Electron microscopic evidence for externalization of the transferrin receptor in vesicular form in sheep reticulocytes. J Cell Biol 1985;101:942-8.

9. Henderson MC, Azorsa DO. The genomic and proteomic content of cancer cell-derived exosomes. Front Oncol 2012;2:38.

10. Franzen CA, Blackwell RH, Foreman KE, et al. Urinary Exosomes: The Potential for Biomarker Utility, Intercellular Signaling and Therapeutics in Urological Malignancy. J Urol 2016;195:1331-9.

11. Rabinowits G, Gerçel-Taylor C, Day JM, et al. Exosomal microRNA: a diagnostic marker for lung cancer. Clin Lung Cancer 2009;10:42-6.

12. Kharaziha P, Ceder S, Li Q, et al. Tumor cell-derived exosomes: a message in a bottle. Biochim Biophys Acta 2012;1826:103-11.

13. Simpson RJ, Lim JW, Moritz RL, et al. Exosomes: proteomic insights and diagnostic potential. Expert Rev Proteomics 2009;6:267-83.

14. Dear JW, Street JM, Bailey MA. Urinary exosomes: a reservoir for biomarker discovery and potential mediators of intrarenal signalling. Proteomics 2013;13:1572-80.

15. Gámez-Valero A, Lozano-Ramos SI, Bancu I, et al. Urinary extracellular vesicles as source of biomarkers in kidney diseases. Front Immunol 2015;6:6. 
16. Bourderioux M, Nguyen-Khoa T, Chhuon C, et al. A new workflow for proteomic analysis of urinary exosomes and assessment in cystinuria patients. J Proteome Res 2015;14:567-77.

17. Bruschi M, Ravera S, Santucci L, et al. The human urinary exosome as a potential metabolic effector cargo. Expert Rev Proteomics 2015;12:425-32.

18. Hurst RE. Does the biomarker search paradigm need rebooting? BMC Urol 2009;9:1.

19. Panfoli I, Ravera S, Podestà M, et al. Exosomes from human mesenchymal stem cells conduct aerobic metabolism in term and preterm newborn infants. FASEB J 2016;30:1416-24.

20. Bruschi M, Santucci L, Ravera S, et al. Human urinary exosome proteome unveils its aerobic respiratory ability. J Proteomics 2016;136:25-34.

21. Arslan F, Lai RC, Smeets MB, et al. Mesenchymal stem

Cite this article as: Panfoli I. Exosomes as "translational" cancer promoter organelles. Transl Cancer Res 2016;5(S2):S205-S207. doi: $10.21037 /$ tcr.2016.08.08 cell-derived exosomes increase ATP levels, decrease oxidative stress and activate PI3K/Akt pathway to enhance myocardial viability and prevent adverse remodeling after myocardial ischemia/reperfusion injury. Stem Cell Res 2013;10:301-12.

22. Yang L, Wu XH, Wang D, et al. Bladder cancer cellderived exosomes inhibit tumor cell apoptosis and induce cell proliferation in vitro. Mol Med Rep 2013;8:1272-8.

23. Franzen CA, Blackwell RH, Todorovic V, et al. Urothelial cells undergo epithelial-to-mesenchymal transition after exposure to muscle invasive bladder cancer exosomes. Oncogenesis 2015;4:e163.

24. Kondaveeti Y, Guttilla Reed IK, White BA, et al. Epithelial-mesenchymal transition induces similar metabolic alterations in two independent breast cancer cell lines. Cancer Lett 2015;364:44-58. 